\title{
Accuracy of Primary Care Clinicians in Screening for Diabetic Retinopathy Using Single-Image Retinal Photography
}

\author{
Tillman F. Farley, $M D^{1}$ \\ Naresh Mandava, $M D^{2}$ \\ F. Ryan Prall, $M D^{2}$ \\ Cece Carsky, MPH, RD, CDE \\ 'Salud Family Health Centers, Fort Lupton, \\ Colorado \\ ${ }^{2}$ University of Colorado Health Science \\ Center, Rocky Mountain Lions Eye Insti- \\ tute, Aurora \\ ${ }^{3}$ Colorado Community Managed Care \\ Network, Denver
}

$\sqrt{1}$

MORE ONLINE

www.annfammed.org

\begin{abstract}
PURPOSE Diabetic patients with limited access to ophthalmologists have low screening rates for diabetic retinopathy. We evaluated a diabetic retinopathy screening program in a community health center using single images taken with a nonmydriatic retinal camera and primary care clinicians trained to read retinal images.
\end{abstract}

METHODS This study was conducted from 2001 to 2004 in a multisite community health center staffed by family physicians, advanced practice nurses, and physician's assistants. The clinic serves a primarily low-income, Hispanic population. Clinic clinicians were trained to read the retinal photographs. All images were overread by an ophthalmologist. Patients were referred to eye care specialists for severe diabetic retinopathy, unknown or other abnormality, or inadequate photographs. We analyzed agreement between the clinicians and the ophthalmologist in recognizing diabetic retinopathy and in determining which patients needed referral. We also analyzed overall screening rates based on clinic access to the camera.

RESULTS One thousand forty diabetic patients were screened for diabetic retinopathy at the health center. One hundred thirteen $(10.9 \%)$ were found to have diabetic retinopathy, 46 severe enough to warrant referral to an ophthalmologist. The clinicians failed to refer 35 (10.2\%) of the 344 patients the ophthalmologist believed needed referral. Most cases of missed referral were due to failure to recognize an inadequate photograph or for abnormalities other than diabetic retinopathy. Screening rates were better in the clinic with a permanent camera.

CONCLUSIONS Primary care clinicians trained to read single images from a retinal camera have acceptable accuracy in screening for diabetic retinopathy. Further training may be necessary to recognize other common abnormalities.

Ann Fam Med 2008;6:428-434. DOI: 10.1370/afm.857.

\section{INTRODUCTION}

$\mathrm{D}$ iabetic retinopathy is the leading cause of acquired blindness in the United States in patients aged 20 to 74 years. ${ }^{1-3}$ Studies show that earlier recognition of diabetes, better glucose control, and early detection and treatment of diabetic retinopathy can slow or prevent the development of blindness. ${ }^{4,5}$ Vulnerable populations, including minorities and the elderly, are disproportionately affected by diabetes. ${ }^{6}$ Moreover, because these groups are more likely to be uninsured or underinsured, they have less access to medical care and are therefore disproportionately affected by the complications of diabetes, including diabetic retinopathy.-9

Effective screening and treatment programs can greatly reduce the burden of blindness in vulnerable populations. ${ }^{10}$ Screening for diabetic retinopathy in vulnerable patients is difficult to achieve, however, it has historically depended on access to specialist physicians by a patient 
population that often does not even have access to primary care physicians. ${ }^{11-13}$ Among minorities and other populations with limited access to specialty medical care, screening rates are generally in the range of $10 \%$ to $20 \%{ }^{14-18}$ In such settings, poor adherence to retinal examination guidelines may reflect a system that selectively denies specialist care to poor and uninsured people, rather than a lack of attention or knowledge on the part of the primary care physician.

The current standard of care for screening for diabetic retinopathy is either a dilated eye examination performed by an ophthalmologist or dilated ETDRS (Early Treatment Diabetic Retinopathy Study) 7-standard field stereoscopic $30^{\circ}$ fundus photography. ${ }^{19}$ Numerous studies have also shown a favorable comparison between single-image retinal cameras and the standard screening methods recommended by the American Diabetes Association (ADA). ${ }^{20-24}$ These cameras take high-quality, $45^{\circ}$ retinal photographs, usually without pupil dilation. Camera operation does not require a highly trained technician, and retinal photographs can be taken quickly and easily in a primary care office, using digital technology or Polaroid film. At least 1 study has suggested that single photographs are inadequate for detecting diabetic retinopathy, but this study included the occurrence of any diabetic retinopathy, including mild diabetic retinopathy not requiring referral. ${ }^{25}$ Studies cited above have shown that the sensitivity of the single-photograph camera in detecting moderate to severe nonproliferative and proliferative diabetic retinopathy is greater than $90 \%$.

We describe a screening program for diabetic retinopathy using single-field nonmydratic retinal photography and primary care clinicians trained to read the photographic images. The study took place in a community health center serving low-income patients in northern Colorado. Most diabetic patients at the clinic were uninsured. Because of the rural location of several clinics, the lack of area eye specialists who accept uninsured patients, and the low rate of private insurance, few diabetic patients managed by this clinic system had access to specialist eye care. Screening rates for diabetic retinopathy in this population have historically been less than $10 \%$.

Our study assessed primary care clinicians' accuracy in reading the single-image retinal photographs and in correctly determining which patients need referral. We also secondarily assessed whether screening rates varied with the access model to the retinal camera. We did not assess the accuracy of the camera itself against the more traditional means of screening for diabetic retinopathy, nor did we assess results from any actual referrals made.
This study was funded by the American Diabetes Association Lions/Sightsavers Research Grant, and the research project was approved by the Colorado Multiinstitutional Review Board.

\section{METHODS}

Informed consent was obtained from all participants in the study. The study was implemented in a multisite federally qualified migrant/community health center serving a large part of northern and northeastern Colorado. The study clinics were staffed full time by family physicians, physician's assistants, and nurse-practitioners. Ninety-five percent of health center patients lived below $200 \%$ of the federal poverty line, and $67 \%$ lived below $100 \%$ of the federal poverty line. Sixty-five percent of clinic patients identified themselves as Hispanic. Sixty percent of the patients were completely uninsured, $28 \%$ were insured through Medicaid, $6 \%$ were covered under Medicare, and $6 \%$ had private insurance.

Six health center clinics participated in the study. Two nonmydriatic retinal cameras (Canon Non-Mydriatic Retinal Camera with Polaroid Back, Canon USA, Lake Success, New York) were assigned to the 6 clinics. One camera was placed permanently at a large clinic (clinic 1 , or $\mathrm{C} 1$ ), and another traveled among 3 other clinics $(\mathrm{C} 2, \mathrm{C} 3$, and $\mathrm{C} 4)$. The final 2 clinics $(\mathrm{C} 5$, C6) were required to refer their patients to $\mathrm{C} 1, \mathrm{C} 2$, $\mathrm{C} 3$, or $\mathrm{C} 4$ for screening.

Diabetic patients eligible for the study had single photographs taken of each retina. Photographs were initially read by a primary care clinician trained in reading images from the retinal camera. Results of those readings were entered into a database. All photographs were then overread by a retinal specialist (N.M.) at the University of Colorado. Results were returned to the primary investigator (T.F.) and entered into the database. Finally, patients needing referral (as determined by the retinal specialist) were contacted, and referrals to eye care specialists were made. Depending on the findings, patients were referred either directly to an ophthalmologist or sometimes first to an optometrist. Optometrists were often able to arrange further referral to an ophthalmologist, if needed. We use the term eye care specialist to include both optometrists and ophthalmologists. This study did not assess results of the actual referrals.

All nonpregnant patients older than 18 years with a diagnosis of diabetes were determined to be eligible to have eye photographs taken. Diabetic prenatal patients were excluded because of the high risk of rapidly progressive diabetic retinopathy in that population and the consequent need for immediate referral to an ophthalmologist. 
A variety of methods were used to encourage patients to undergo retinal screening. All clinicians were advised of the program and encouraged to schedule patients for retinal photography. In addition, letters were sent to diabetic patients asking them to make appointments for retinal screening. Finally, in clinics with a camera available, diabetic patients were encouraged to have retinal screening done on a walk-in basis at the end of their clinic visits. No assessment was made of how or with what degree of rigor these various modalities were used in each clinic.

Eight clinicians from the 6 clinics were trained in reading films from the retinal camera. The curriculum for this training was devised by a retinal specialist (N.M.). Because of the lack of access to ophthalmologists by the population under study, we decided to refer only those patients with severe retinopathy (based on the Airlie-House classification system ${ }^{26,27}$ ) needing immediate intervention. Table 1 lists the criteria for referral, as outlined in the training curriculum.

The retinal photographs were taken by specifically trained camera operators. All photographs were taken of undilated eyes. Because we were looking for diabetic retinopathy only, and because an ophthalmologic examination is considered the reference standard of care, patients were informed that the photographs were not meant to take the place of a regular dilated eye examination by an eye-care specialist.

There were 2 main outcome variables in this evaluation. First, we compared the clinicians' reading of each film with the ophthalmologist's reading. Outcomes were analyzed in terms of sensitivity, specificity, and positive and negative predictive values. The denominator for this variable included all diabetic patients screened during the 3 -year course of the program. Second, we determined the screening rates of patients at the various clinics. For this variable, we included only those diabetic patients seen at least 2 times during the study period. Patients were considered to have been

\section{Table 1. Criteria for Referral to Eye Care Specialist}

Uninterpretable (inadequate) photographs

Severe diabetic retinopathy as evidenced by any of the following: Diffuse intraretinal hemorrhage in all 4 quadrants

Any hard lipid exudate present within 1/3 disc diameter of fovea

An optic-disc-sized patch of hard lipid exudate, any part of which is within 1 disc diameter of the fovea

Venous beading present in 2 quadrants

Any intraretinal microvascular abnormalities

Any neovascularization

Any vitreous hemorrhage

Other abnormalities, including retinal scarring or past laser surgery Any unknown abnormalities screened if they had any photographs taken during the 3 years of the study period, regardless of the whether the photographs were interpretable. SAS v 8.0 (SAS Institute, Cary, NC) was used for all data analysis.

Before implementing the program, the curriculum was tested on 20 primary care clinicians. After a single 3-hour training session using the devised curriculum, the 20 clinicians were tested using 100 standardized images of nondialated eyes from 50 diabetic patients at a health center in Colorado. Using the reading from the retinal specialist ophthalmologist (N.M.) as the reference standard, the overall sensitivity and specificity of the 20 clinicians in reading these photographs were $88 \%$ and $92 \%$, respectively.

\section{RESULTS}

During the 3-year course of the study, a total of 1,040 unduplicated diabetic patients had their eyes photographed. Because many patients at this health center are transient and seen only once, we decided to include in the screening rate analysis only those patients making at least 2 visits to the clinic during the 3-year course of the study. Of the 1,040 total patients screened, 96 made only 1 visit to the clinic system during that 3 -year period, leaving 944 who met criteria for inclusion in the screening rate portion of the study. A total of 2,586 diabetic patients made at least 2 visits to the participating clinics during the 3 -year course of the study, for an overall screening rate of $37 \%$ (944 of 2,586). Most, $75 \%$, of the diabetic patients had no insurance, $6 \%$ were insured through Medicaid, 9\% were covered under Medicare, and 10\% had private insurance.

To determine agreement in reading between the primary care clinicians and the retinal specialist, we analyzed findings from all 1,040 patients who were photographed. The retinal specialist determined that in $302(29 \%)$ of the patients, 1 or both of the photographs were not adequate for complete interpretation. The clinicians identified 296 patients $(28 \%)$ as requiring referral for inadequate photographs; these clinicians failed to recognize an inadequate photograph in 39 patients, though 21 of these patients were referred for other reasons. An additional 33 patients the ophthalmologist judged to have adequate photographs were referred by the clinicians for having inadequate photographs. Most common reasons for inadequate photographs included poor camera technique, dirty optics, overly small pupils, or the presence of dense cataracts. Clinicians tended to overinterpret photographs that the retinal specialist thought were too dark (underexposed). If adequate photographs could not be obtained, referral was made to eye care specialists. 
A total of 113 of 1,040 patients (10.9\%) were determined by the retinal specialist to have some degree of diabetic retinopathy. Forty-six of these 113 patients $(40.7 \%)$ had diabetic retinopathy severe enough to warrant referral. Thirteen of the patients referred for severe diabetic retinopathy were found among the 302 patients also determined to have inadequate photographs (in these patients, the photographs did not meet the quality standards set in the protocol but nevertheless showed enough evidence of severe diabetic retinopathy to warrant referral on that basis as well). The remaining 33 patients with severe diabetic retinopathy were found among the 738 patients with adequate photographs. The overall rate of severe diabetic retinopathy was $4.4 \%$ ( 46 of 1,040 ). This estimate is likely low given that patients with inadequate photographs may be more likely to have diabetic retinopathy.

The primary care clinicians showed reasonable accuracy in recognizing important diabetic retinopathy. Overall, these clinicians failed to recognize severe diabetic retinopathy in 5 of the 46 patients determined to have it by the ophthalmologist. In 1 of these patients, the clinician recommended referral for other reasons (inadequate photograph), which left 4 missed referrals out of the 46 patients with severe diabetic retinopathy ( $91 \%$ sensitivity, $99 \%$ specificity). Of the 69 patients that clinicians referred for severe diabetic retinopathy, the retinal specialist agreed with the reason for referral in 41 patients (positive predictive value, $59.4 \%$; negative predictive value, $97 \%$ ). All results are displayed in Table 2.
Sixty-one patients of 1,040 (5.9\%) patients were found by the retinal specialist to have other abnormalities, 39 of whom required referral $(3.75 \%$ of 1,040$)$. The most common other abnormality was an increased cup-to-disc ratio. Other abnormalities also included choroid nevus, arterial plaque, severe arterial attenuation and tortuosity, drusen, arterial sclerosis, macular degeneration, myelinated nerve fiber layer, choroidal atrophy, small optic nerve, epiretinal membrane, lipemia retinalis, and idiopathic perifoveal telangiectasia. Many of these findings were recognized as abnormal by the study clinicians; however, they failed to recognize 13 of the other abnormalities as requiring referral. Reasons for all missed referrals are listed in Table 3.

Screening rates varied significantly according to access model, with the clinic with the permanent camera having the highest screening rate (Table 4). When compared with the clinic with the permanent camera, all but 1 of the other clinics had a significantly lower screening rate.

Overall costs of the program included approximately $\$ 25,000$ for each of the 2 cameras, plus approximately $\$ 30,000$ per year for each of 2 the trained camera operators (1 camera operator per camera). In addition, each primary care clinician involved in the study lost a halfday of clinic productivity during training.

\section{DISCUSSION}

Our study showed that primary care clinicians can be trained to recognize diabetic retinopathy using

Table 2. Results of Photograph Interpretations $(n=1,040)$ by Primary Care Clinician and Ophthalmologist

\begin{tabular}{|c|c|c|c|c|c|c|}
\hline Reading Outcome, All Patients & Ophthalmologist & $\begin{array}{c}\text { Primary Care } \\
\text { Clinician }\end{array}$ & Sensitivity & Specificity & PPV & NPV \\
\hline Diabetic retinopathy, overalla & 113 & 156 & 0.85 & 0.94 & 0.62 & 0.98 \\
\hline Vitreous hemorrhage & 5 & 7 & 1.0 & 0.997 & 0.71 & 1.0 \\
\hline Intraretinal microvascular abnormality & 5 & 3 & 0.2 & 0.998 & 0.33 & 0.996 \\
\hline Venous beading & 6 & 11 & 0.50 & 0.995 & 0.27 & 0.997 \\
\hline 2 quadrants & 4 & 9 & 0.50 & 0.99 & 0.22 & 0.99 \\
\hline Intraretinal hemorrhage & 98 & 90 & 0.73 & 0.98 & 0.80 & 0.97 \\
\hline Diffuse & 4 & 6 & 0.75 & 0.99 & 0.50 & 0.99 \\
\hline Neovascularization & 5 & 4 & 0.4 & 0.997 & 0.50 & 0.998 \\
\hline Lipid exudates & 56 & 108 & 0.96 & 0.95 & 0.50 & 0.99 \\
\hline $1 / 3$ disc diameter & 39 & 52 & 0.82 & 0.98 & 0.62 & 0.99 \\
\hline Disc diameter & 16 & 29 & 0.94 & 0.99 & 0.52 & 0.99 \\
\hline Referred for other abnormality ${ }^{b}$ & 39 & 26 & 0.66 & 0.97 & 0.38 & 0.96 \\
\hline Referred for diabetic retinopathy & 46 & 69 & 0.89 & 0.97 & 0.59 & 0.97 \\
\hline Referred for inadequate photograph & 302 & 296 & 0.87 & 0.96 & 0.89 & 0.95 \\
\hline Referred, totalc & 344 & 356 & 0.90 & 0.93 & 0.87 & 0.95 \\
\hline
\end{tabular}


nonmydriatic photography with a reasonable degree of accuracy. The overall missed referral rate for all screened patients was $3.3 \%$ (35 of 1,040). Overall, the clinicians missed $10.2 \%$ (35 of 344 ) of the referrals that the ophthalmologist deemed necessary. The clinicians correctly identified the other 309 patients needing referral. The overall sensitivity for the primary care clinicians' ability to appropriately refer patients was $89.8 \%$. Most of the missed referrals (18 of $35,51.4 \%$ ) were for failure to recognize an inadequate photograph. Small pupils, poor photographic technique, and dirty optics accounted for most of the inadequate photographs. Although we did not track these data, it is possible that patients with an inadequate photograph would have a higher prevalence of severe diabetic retinopathy.

Besides failure to recognize inaccurate photographs, the clinicians had the most problems recognizing other abnormalities that were not described in the training curriculum. It may be that by expanding the scope of the curriculum, these clinicians could also adequately recognize such abnormalities as an enlarged

\section{Table 3. Ophthalmologist Referrals Missed by Primary Care Clinician}

\begin{tabular}{lc}
\hline Abnormality Missed & No./Denominator ${ }^{\text {a }}$ (\%) \\
\hline Inadequate photograph & $18 / 302(6.0)$ \\
Other abnormality needing referral (total) & $13 / 39(33.3)$ \\
Abnormal cup-to-disc ratio & 5 \\
Severe arterial attenuation and tortuosity & 1 \\
Unknown abnormality & 3 \\
Choroidal nevus & 2 \\
Small optic nerve & 1 \\
Arterial plaque & 1 \\
Diabetic retinopathy & $4 / 46(8.7)$ \\
Total missed referrals & $35 / 344(10.1)$ \\
\hline
\end{tabular}

a Denominator is the number of referrals for each category made by the retinal specialist.

${ }^{b}$ Clinicians missed severe diabetic retinopathy in 5 cases; 1 patient was referred for inadequate photograph and does not count as missed referral.

Table 4. Patient Screening Rates by Clinic Access to Retinal Camera

\begin{tabular}{|c|c|c|c|c|}
\hline $\begin{array}{l}\text { Clinic } \\
\text { (Access Model) }\end{array}$ & $\begin{array}{c}\text { No. } \\
\text { Screened }\end{array}$ & $\begin{array}{c}\text { No. of Patients } \\
\text { With Diabetes } \\
\text { Mellitus }\end{array}$ & $\begin{array}{l}\text { Screening } \\
\text { Rate }\end{array}$ & $P$ Value $^{a}$ \\
\hline C1 (permanent) & 314 & 673 & 47 & NA \\
\hline C2 (twice yearly) & 261 & 587 & 44 & .436 \\
\hline C3 (twice yearly) & 114 & 297 & 38 & .017 \\
\hline C4 (twice yearly) & 130 & 519 & 25 & $<.001$ \\
\hline C5 (refer in) & 95 & 368 & 26 & $<.001$ \\
\hline C6 (refer in) & 30 & 142 & 21 & $<.001$ \\
\hline Total & 944 & 2,586 & 37 & $<.001$ \\
\hline
\end{tabular}

cup-to-disc ratio and choroidal nevi. It would be difficult to develop a curriculum that could address all of the myriad abnormalities found in retinal examinations unrelated to diabetic retinopathy.

As might be expected, the clinicians were most accurate in recognizing abnormalities of diabetic retinopathy that occur with the highest frequency (lipid exudates and intraretinal hemorrhage) and that are the most striking in appearance (vitreous hemorrhage). The clinicians were much less sensitive in recognizing subtle and unusual findings, such as venous beading and intraretinal microvascular abnormalities. These abnormalities occurred with a low frequency in the population studied. Supplemental Figures 1 and 2 (available online at http://www.annfammed.org/ cgi/content/full/6/5/428/DC1) illustrate important diabetic retinopathy missed by the clinicians.

Our study also showed that reasonable screening rates for diabetic retinopathy among a low-income population can be achieved using single-image retinal photography. Screening rates were highest in the clinic with a permanent camera, although they were still less than targeted standards. This low rate is likely partly due to the transient nature of the population served by this clinic system. It's possible this rate could be improved using a more aggressive protocol to get patients in for screening. Clinics with a camera available twice yearly varied substantially in their screening rates. Interviews with clinicians at those clinics suggested that the intermittent nature of camera availability made it less likely for them to remember to refer patients for retinal photography. Clinics referring patients to another clinic for screening had the lowest screening rates. The wide variation in screening rates are likely due to differences in how clinicians at individual clinics chose to refer patients for screening; some clinics were more efficient than others.

Other studies have shown that single-image retinal photography offer a reasonable alternative for screening for diabetic retinopathy. ${ }^{20-24}$ At least 1 study found that family physicians can accurately detect diabetic retinopathy using a nonmydriatic ophthalmoscope. ${ }^{28}$ Another study found reasonable accuracy when automated image analysis was applied to nonmydriatic photographs. ${ }^{29}$ Our study is the first to assess both an 
access model for diabetic retinopathy screening using a single image taken with a retinal camera and primary care clinicians trained to read the images.

There are a number of limitations to this study. Most importantly, the type of photographic examination undertaken in this study does not attempt to take the place of a more comprehensive examination by an eye care specialist. We were exploring a new way to screen for diabetic retinopathy in primary care. All patients in the study were advised that they should try to obtain care from an eye care specialist. For financial reasons, this option was not available to the great majority of our patients.

Second, we had many inadequate photographs. For the purposes of the study, we did not use mydriatic agents, which greatly limited our ability to obtain good photographs in patients with small pupils. Since the end of the study period, we have been using mydriatic drops as needed, and our inadequate photograph rate is now less than $1 \%$. Our data would have been easier to interpret had we implemented this procedure as part of the research study.

A third limitation to our study is the nature of the patient population in our community health center. Because many patients drift through the area or go through cycles of losing and regaining health insurance, they are seen intermittently. For that reason, we decided not to measure our success rate based on the recommendation of yearly screening; instead, we based it on any screening examination done during the 3 year course of the study. We recognize that measure does not conform to the screening standards set by the American Diabetes Association, US Public Services Task Force, and others; however, the efficacy of annual screening compared with another interval has not been well established.

There are limitations as to the questions our study can answer. We did not attempt to evaluate the retinal camera itself beyond noting the high numbers of inadequate photographs when not using mydriasis, nor did we attempt to assess the reading proficiency of individual clinicians. There may have been wide variations in skill among clinicians based on the number of photographs read or based on the access model at each clinician's clinic. Our study did not attempt to look at how successful we were in making referrals to eye care specialists or the interventions resulting from those referrals. Our health care system extends more than 15,000 square miles. Each clinic has its own referral network. Finding specialty care for our patients is difficult under any circumstance and depends on any number of variables specific to individual clinics and patient circumstances. Furthermore, because we did not have prestudy rates of screening with which to compare our study rates, we cannot definitively say that our screening rates improved with this program. Finally, we did not standardize the procedures used by the various clinics to get patients in for screening. Some clinics were fairly aggressive in sending out reminder cards, making telephone calls, and using the no-missed-opportunities approach, whereas other clinics were less so, which likely explains the wide variation in screening rates among the clinics without a permanent camera.

In the final analysis, we decreased the number of patients needing referral to eye care specialists for diabetic retinopathy from 1,040 to 344 . Our screening rate for diabetic retinopathy, at least in the clinic with a permanent camera, compared favorably with national data for insured populations. The primary care clinicians in this study missed important diabetic retinopathy in $8.7 \%$ of the patients who had it (4 of 46 ), but discovered retinopathy in 41 others (with 1 patient with severe diabetic retinopathy referred for an inadequate photograph). Because of the many uninsured and low-income diabetic patients in our system, we could easily overwhelm any eye care specialist who agreed to see them for screening and treatment. We believe that by taking on the screening burden ourselves, local eye care specialists may be more willing to see the far fewer patients with retinopathy needing further evaluation and treatment. With this program, we believe that we will be able to obtain specialty care for our patients with severe diabetic retinopathy before it causes vision loss.

Using single-image retinal photographs taken with a nonmydriatic retinal camera in primary care offices, with primary care clinicians reading the resulting images, may be a cost-effective way to help reduce vision loss in diabetic patients who have limited access to ophthalmologists.

To read or post commentaries in response to this article, see it online at http://www.annfammed.org/cgi/content/full/6/5/428.

Key words: Diabetic retinopathy; mass screening; low income population; primary health care; single photograph retinal camera

Submitted September 25, 2007; submitted, revised, January 30, 2008; accepted February 12, 2008.

Versions of this report were presented as follows: Diabetic Retinopathy: Screening in Primary Care, presented at the Best Practices Forum, National Association of Community Health Centers, Lake Tahoe, Nevada, February, 2002; Screening for Diabetic Retinopathy in Primary Care, North American Primary Care Research Group 32nd Annual Meeting, Banff, Alberta, Canada, October, 2003; and Screening for Diabetic Retinopathy in Farmworker Populations, 2005 National Farmworker Health Conference, San Juan, Puerto Rico. May 13, 2005.

Funding support: This study was supported by a Lion's SightFirst Diabetic Retinopathy Research grant, 2001-2004. 
Disclaimer: This article is not in any way meant to serve as an endorsement for any particular manufacturer of ophthalmologic equipment.

Acknowledgments: We would like to thank the American Diabetes Foundation Lions Sightsaving Research Grant Program for funding this study. We would also like to thank Stanley J. Brasher, the CEO of Salud Family Health Centers since 1972, for a lifetime commitment to improving the health and lives of the immigrant and low-income populations of Colorado and New Mexico. Without his vision and his support of innovative programs, this study would not have been possible.

\section{References}

1. Harris MI. Diabetes in America: epidemiology and scope of the problem. Diabetes Care. 1998;21(suppl 3):C11-C14.

2. Mokdad AH, Ford ES, Bowman BA, et al. Diabetes trends in the US: 1990-i998. Diabetes Care. 2000;23(9):1278-1283.

3. Varma R. Diabetic retinopathy: challenges and future directions. Am J Ophthalmol. 2006;141(3):539-541.

4. The effect of intensive treatment of diabetes on the development and progression of long-term complications in insulin-dependent diabetes mellitus: the Diabetes Control and Complications Research Group. N Engl J Med. 1993;329(14):977-986.

5. Intensive blood glucose control with sulphonylureas or insulin compared with conventional treatment and risk of complications in patients with Type II diabetes. Lancet. 1998;352(9178):839-855.

6. Harris MI, Flegal KM, Cowie CC, et al. Prevalence of diabetes impaired fasting glucose, and impaired glucose tolerance in US adults: the 3rd National Health and Nutrition Survey, 1988094 Diabetes Care. 1998;21(4):518-524.

7. Wong TY, Klein R, Islam FM, et al. Diabetic retinopathy in a multi-ethnic cohort in the United States. Am J Ophthalmol. 2006;141(3):446-455.

8. Varma R, Torres M, Pena F, Klein R, Azen SP. Los Angeles Latino Eye Study Group. Prevalence of diabetic retinopathy in adult Latinos: the Los Angeles Eye Study. Ophthalmology. 2004;111(7):1298-1306.

9. Pugh JA, Tuley MR, Hazuda HP, Stern MP. Influence of outpatient insurance coverage on the microvascular complications of non-insulin dependent diabetes in Mexican-Americans. J Diabetes Complications. 1992;6(4)236-241.

10. Ferris FL III. How effective are treatments for diabetic retinopathy? JAMA. 1993;269(10):1290-1291.

11. Lewin-Epstein N. Determinants of regular source of health care in Black, Mexican, Puerto Rican, and non-Hispanic white populations. Med Care. 1991;29(6):543-557.

12. Valdez RB, Morgenstern R, Brown R, Wyn R, Wang C, Cumberland $W$. Insuring Latinos against the costs of illness. JAMA 1993;269(7):889-894.

13. Moss SE, Klein R, Klein BE. Factors associated with having eye examinations in persons with diabetes. Arch Fam Med. 1995;4(6):529-534
14. Harris MI, Klein R, Cowie CC, Rowland M, Byrd-Holt DD. Is the risk of diabetic retinopathy greater in non-Hispanic Blacks and MexicanAmericans than in non-Hispanic whites with type 2 diabetes? A US population study. Diabetes Care. 1998;21(8):1230-1235.

15. Okada S, Ichiki K, Tanokuchi S, Hamada H, Matsuo N, Ota Z. Factors related to the development and progression of diabetic retinopathy in patients with type 2 diabetes. J Int Med Res. 1996;24(2):214-220.

16. Appiah AP, Ganthier R Jr, Watkins N. Delayed diagnosis of diabetic retinopathy in black and Hispanic patients with diabetes mellitus. Ann Ophthalmol. 1991;23(4):156-158.

17. Taylor CR, Merin LM, Salunga AM, et al. Improving diabetic retinopathy screening ratios using telemedicine-based digital retinal imaging technology: the Vine Hill Study. Diabetes Care. 2007;30(3):574-578

18. Will JC, German RR, Schuman E, Michael S, Kurth DM, Deeb L. Patient adherence to guidelines for diabetes eye care: results from the diabetic eye disease follow up study. Am J Public Health. 1994;84(10):1669-1671.

19. American Academy of Ophthalmology Retina Panel. Preferred Prac tice Patterns Committee. Diabetic Retinopathy. San Francisco, CA: American Academy of Ophthalmology; 1998.

20. Higgs ER. Harney BA, Kelleher A, Reckless JP. Detection of diabetic retinopathy in the community using a single photograph retinal camera. Diabet Med. 1991;8(6):551-555.

21. Buxton MJ, Sculpher MJ, Ferguson BA, et al. Screening for treatable diabetic retinopathy: a comparison of different methods. Diabet Med. 1991;8(6):371-377.

22. Chantelau E, Zwecker M, Weiss H, Kluxen G, Berger M. Fundus polaroid screening for diabetic retinopathy. Is one print per patient enough? Diabetes Care. 1989;12(3):223-226.

23. Taylor R. Practical community screening for diabetic retinopathy using the mobile single photograph retinal camera: a report of a 12 centre study. Diabet Med. 1996;13(11):946-952.

24. Williams GA, Scott I, Haller JA, Maguire AM, Marcus D, McDonald HR. Single-field fundus photography for diabetic retinopathy screening: a report by the American Academy of Ophthalmology. Ophthalmology. 2004;111(5):1055-1062.

25. Kuo HK, Hsieh HH, Liu RT. Screening for diabetic retinopathy by one-field, non-mydriatic, 45 degrees digital photography is inadequate. Ophthalmologica. 2005;219(5):292-296.

26. Grading diabetic retinopathy from stereoscopic color fundus photographs: an extension of the modified Airlie House classification. ETDRS report 10. Ophthalmology. 1991;98(5 Suppl):786-806.

27. The Diabetic Retinopathy Study Research Group. A modification of the Airlie House classification of diabetic retinopathy. Invest Ophthalmol Vis Sci. 1981;21(2):210-226.

28. Gill JM, Cole DM, Lebowitz HM, Diamond JJ. Accuracy of screening for diabetic retinopathy by family physicians. Ann Fam Med. 2004;2(3):218-220.

29. Hansen AB, Hartvig NV, Jensen MS, Borch-Johnson K, Lund-Andersen $\mathrm{H}$, Larsen $\mathrm{M}$. Diabetic retinopathy screening using digital nonmydriatic fundus photography and automated image analysis. Acta Ophthalmol Scand. 2004;82(6):666-672. 